\title{
ELECTRONIC TRANSPORT IN MO/Ni SUPERLATTICES
}

Roy Clarke, Donald Morelli and Ctirad Uher

Department of Physics, The University of Michigan, Ann Arbor, MI 48109

and

Hitoshi Homma and Ivan K. Schuller

Materials Science and Technology Division, Argonne National Laboratory, Argonne IL 60439

(Received 13 . August 1984 by John D Dow)

An experimental relationship between superconductivity, magnetism and localization is explored in short-wavelength ( $14 \AA \leqslant \Lambda<40 \AA$ ) sputtered $\mathrm{Mo} / \mathrm{Ni}$ superlattices. A crossover to a superconducting state is observed for $\Lambda<9 \AA$ consistent with the observed paramagnetic behavior when the $\mathrm{Ni}$ strata are four atomic layers thick, or less. All samples show localization effects at helium temperatures and non-superconducting samples develop an unusual resistance plateau below $T \simeq 0.5 \mathrm{~K}$.

Artificial modulated heterostructures provide many interesting examples of physical phenomena that are difficult to realize in any other way. The major emphasis so far has been technological, directed at semiconducting superlattices based primarily on GaAs-GaAlAs ${ }^{\perp}$. However, the broader class of novel

heterostructures, particularly metallic superlattices, are also of great interest for fundamental studies of collective effects in the restricted geometry of thin layers and interfaces.

In this paper we present the results of a combined study of the structural and electronic properties of Mo/ $\mathrm{Ni}$ superlattices grown by the sequential sputtering technique ${ }^{2}$. We focus on the region of short modulation wavelength ( $14 \AA<\Lambda<40 \AA$ ) where strong departures from normal bulk properties may be expected. In our experiments these are most clearly seen in the competition between magnetic ( $\mathrm{Ni}$ and superconducting (Mo) behavior, and in the occurrence of localization effects at low temperatures. Although the samples show a surprisingly high degree of stacking coherence normal to the layers there is an intrinsic interfacial mismatch in these mixed bcc-fcc microstructures which is crucial to understanding their electrical properties.

The superlattices were co-sputtered on $90^{\circ}$ sapphire substrates $\left(-1 \mathrm{~cm}^{2}\right.$ area) held at a temperature of $20^{\circ} \mathrm{C}^{3}$. The total thickness of the superlattices was approximately $1 \mu \mathrm{m}$. Standard photolithographic techniques were used to etch out a four-point bridge pattern suitable for in-plane $D C$ resistance measurements. The substrates were placed directly inside the mixing chamber of a dilution refrigerator. Electrical contacts were made by uitrasonic soldering with pure indium, and voltages were sensed with a precision of 1 part in $10^{5}$ with excitation currents in the range 10-100 $\mu \mathrm{A}$. $x$-ray characterizations of the superlattices were carried out at room temperature on a four-circle diffractometer. Both $\theta-2 \theta$ and $w$ (rocking) profiles were obtained. The former scans determine the degree of ordering perpendicular to the layers and the latter probe undulations of the layers. In all cases there is polycrystalline texture within the layers ${ }^{3}$.

Figures 1 and 2 show the behavior of the in-plane resistance of various $1: 1$ and $3: 1$ Mo/Ni samples in the small wavelength region. Several unexpected findings are evident: firstly, we note that structures with $\mathrm{Ni}$ layer thickness $<9 \AA$, i.e. less than nominally four atomic layers, show a sharp superconducting transition (see inset of Fig. 1). Referring now to Fig. 2, which shows the corresponding resistance behavior for several longer wavelengths, no transition is observed down to $15 \mathrm{mk}$ on structures with $\mathrm{Ni}$ layers thicker than 9A. This observation of a crossover to superconducting behavior is interpreted as an unequivocal signature of the loss of ferromagnetic order. Indeed, direct measurements of the magnetization, $M_{s}$, as a function of $\Lambda$ show that $M_{S}$ approaches zero at a nickel thickness of $9 A^{4}$ (see Fig. 3). It is tempting to ascribe such behavior to so-called "dead" layers of $\mathrm{Ni}$, however, first one must inquire into the nature of the interface between $\mathrm{Mo}$ and $\mathrm{Ni}$ layers and consider the possibility that alloys are formed which may be nonmagnetic. This question becomes particularly relevant here since we are dealing with the interface of two distinct morphologies: bcc Mo(110) and fcc Ni(111). 


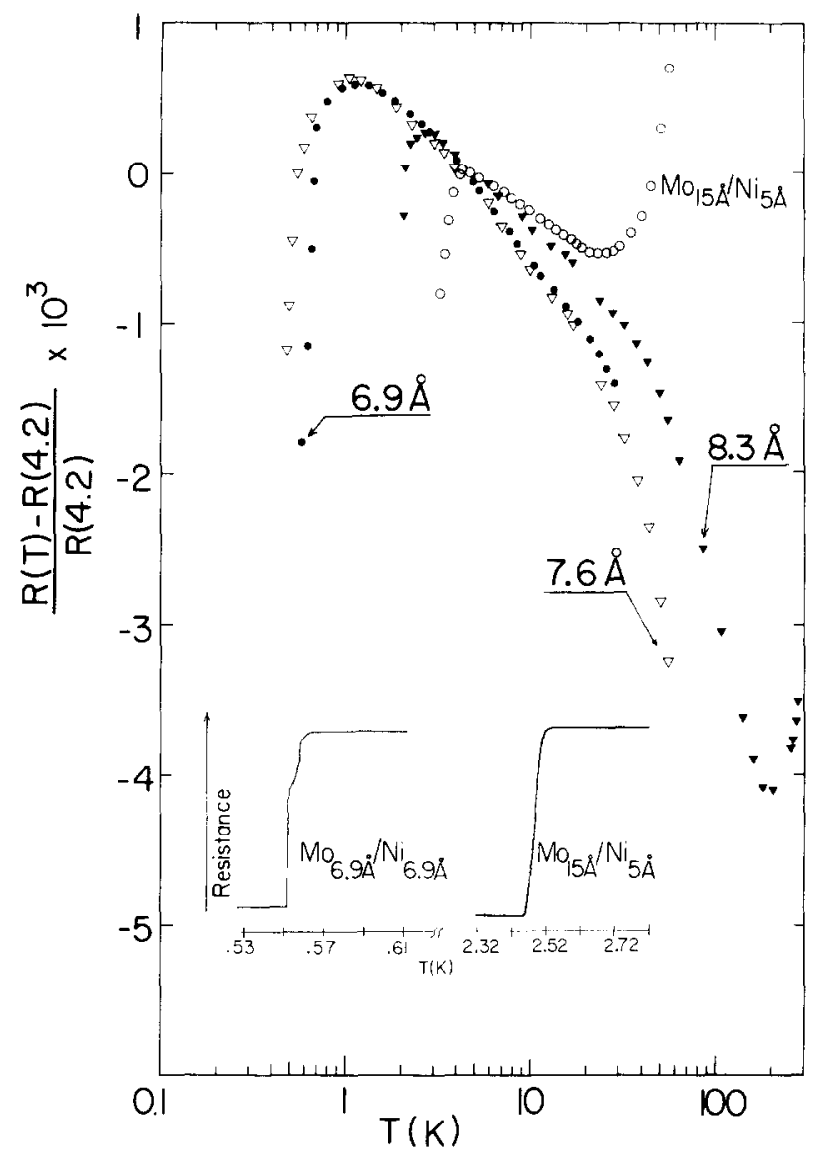

Fig. 1 In-plane resistance, normalized to values at $4.2 \mathrm{~K}$. The labels refer to $\Lambda / 2$ for samples with equal numbers of

Figure 4 compares $X$-ray scattering profiles of three different samples spanning the crossover to superconducting behavior. The two scans shown in the main body of Fig. 4 consist of a sharp principal peak flanked by satellites at $\pm 2 \pi n / \Lambda$, from which the modulation wavelength can be accurately determined. The position of the principal peak gives an average d spacing of $2.14 \AA$ consistent with Mo(110)/Ni(111) stacking. The inverse width of the peaks can be used to determine the coherence length of the ordering perpendicular to the layers. The samples $\Lambda / 2=11.7 \AA$ (nonsuperconducting) and Mo15A/Ni5A

(superconducting) have coherence lengths of at least 250 and $100 \AA$, respectively. Detailed modeling ${ }^{3}$ shows that intermixing occurs at most on one interfacial atomic plane, confirming that for both of these samples the interfacial region has well defined layering with few faults, i.e., at most one every $5 \Lambda$. The layering is further characterized by measurements of the vertical mosaic $\left(-8^{\circ}\right.$ half width at half maximum) which probe
Mo and $\mathrm{Ni}$ layers. The inset shows the sharpness of the transition. angular undulations of the layers across the sample (see Fig. 4, inset (a)). Also, we can rule out interdiffusion at $300 \mathrm{~K}$ since the satelitite intensities do not change over a period of many months.

If both Mo and $\mathrm{Ni}$ constituent layers are made very thin an interesting structural effect is observed. In Fig. 4, inset (b), we see that the diffraction profile for $\Lambda / 2=6.9 \AA$ takes on a broad continuum form reminiscent of an amorphous structure. The short-range order is calculated to extend over only $-20 \mathrm{~A}$ for this sample. Thus, in the small wavelength limit $\Lambda / 2 \leqslant 8 A$, coherence is lost and the samples are more akin to metallic glasses than superlattices. One factor which may be responsible for the loss of coherence is the intrinsic limitation of the sputtering method. Another possibility is that these superlattices cannot sustain more than a critical amount of strain. If this limit is exceeded then it may become more favorable to form a random alloy, as in the $\Lambda / 2=6.9 \AA$ sample shown in Fig. 4 . 


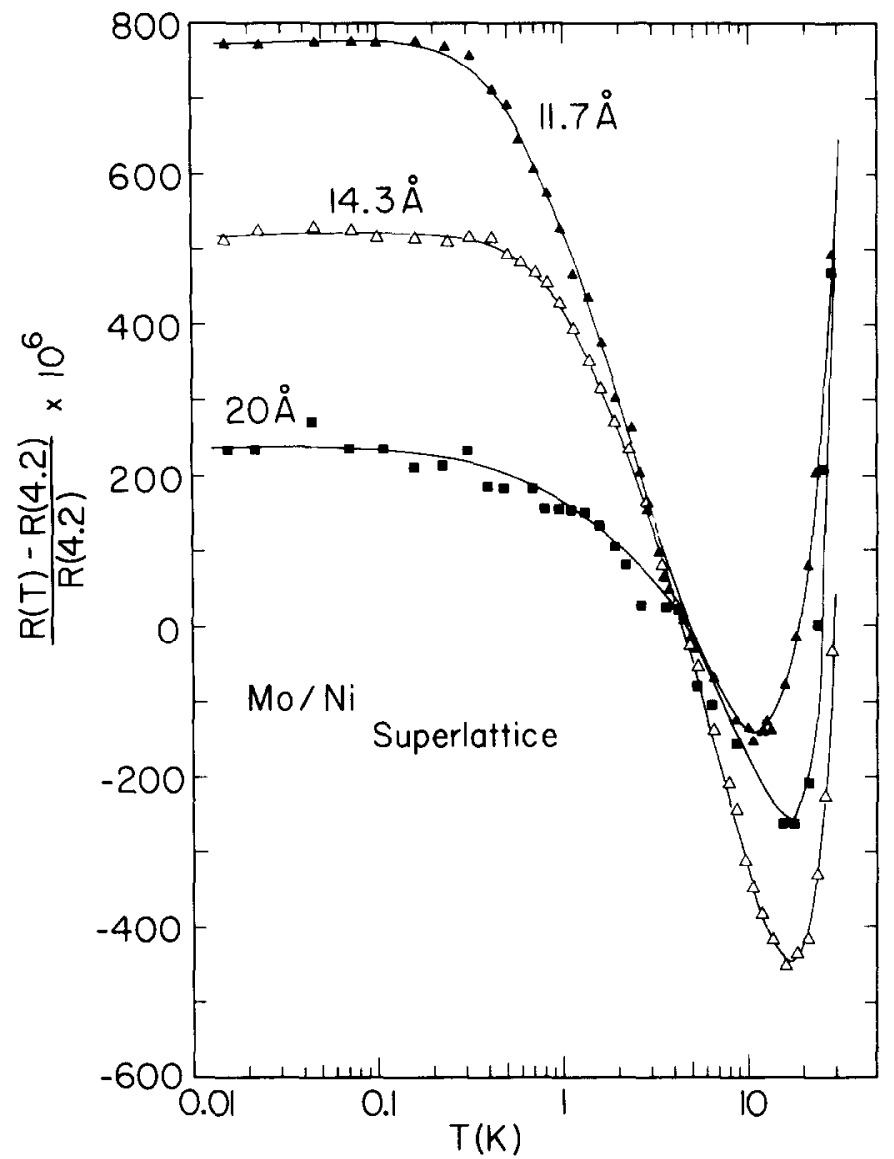

Fig. 2 Temperature dependence of normatized resistance for non-

This has been confirmed by recent molecular dynamics calculations ${ }^{5}$.

To summarize the structural data then, we observe quite coherent layering until the thickness of both constituents is reduced below $-8 A$, in which case a glassy structure is obtained. This structural crossover may be responsible for the grouping of $T_{c}$ 's seen in Fig. 1 i.e., $T_{C}=0.5 K$ for the glassy structures and $T_{C}=2 \mathrm{~K}$ for those with coherent layering and thin $\mathrm{Ni}$ layers. Since the latter temperature is intermediate between the bulk values for crystalline Mo $\left(T_{C}=0.9 \mathrm{~K}\right)$ and amorphous ${ }^{6}$ Mo $\left(T_{C} 9 K\right)$, there is some indication that the short mean free path may be enhancing the $T_{C}$ of Mo, as was recently reported in Mo/Al multilayers.?

As the thickness of the $\mathrm{Ni}$ component is reduced we find that the onset of superconductivity occurs somewhat before the coherence of the layering is lost. This result is suggestive of the existence of magnetically "dead" i.e., paramagnetic, layers when the $\mathrm{Ni}$ strata are less than four atoms thick.
However, we cannot rule out the possibility that a small amount of intermixing could be responsible for this behavior. ${ }^{8}$ The existence of magnetically "dead", i.e., paramagnetic, layers has been discussed extensively in the 7 iterature. The current consensus ${ }^{9-12}$ is that substrate effects are very important. For example, a Ni monolayer is calculated to be ferromagnetic on $\mathrm{Cu}(100)$ but paramagnetic on Cu(111) and Tersoff and Falicov11 concluded that for substrates which couple strongly to the $\mathrm{Ni}$ $f i 1 m$, ferromagnetism is suppressed at around three atomic layers of $\mathrm{Ni}$ (by $\mathrm{sp}-\mathrm{d}$ hybridization); our findings lend support to this conclusion.

Finally, we point out an interesting effect observed on the resistance curves in Fig. 1 and 2 . In all cases, including the superconducting samples, the resistance shows a distinct minimum at low temperatures which deepens and shifts to a higher temperature with decreasing $\Lambda$; in fact, samples with the smallest $\hat{A}(13.8 \AA$ and $15.2 \AA)$ have negative temperature coefficients up to at least room 


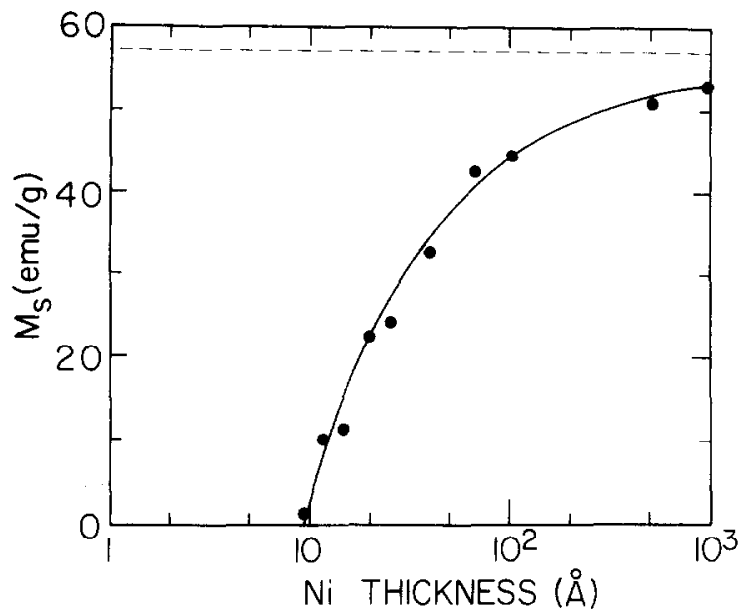

Fig. 3 Saturation magnetization of Mo/Ni structures as a function of Ni thickness. From Ref. [4].

temperature. Absolute resistivities are in the range $60-160 \mu \Omega-\mathrm{cm}$, the actual value being inversely proportional to the modulation wavelength. This fact, coupled with the imperfect vertical mosaic mentioned above, demonstrates the dominance of boundary (interface) scattering in the low-temperature electronic transport. Of particular interest. are the non-superconducting (ferromagnetic) samples, shown in Fig. 2, in which the resistivity levels off in a plateau in the region below $T=0.5 \mathrm{~K}$. Truncation of the resistance rise at low temperatures may signal the importance of finite size effects, such as the effective width of the conduction channel becoming comparable to the inelastic diffusion length. The latter phenomenon was recently searched for in thin, short films of $\mathrm{Au}_{40} \mathrm{Pd}_{60}$ but so far has not been observed ${ }^{3}$. Alternatively, at low enough temperatures the plateau may arise from the destructive influence of strong internal magnetic fields on the spin pairing ${ }^{14}$. It is interesting to note that superconductivity and the existence of the resistivity plateau seem to be mutually exclusive. The reason for this is not presently understood but such effects have been the subject of considerable recent theoretical interest $\mathrm{t}^{15}$. We are now carrying out more detailed experiments to distinguish between the different possible mechanisms and this work will be the subject of a longer publication. In connection with the the resistivity plateau, it is interesting to note that an almost identical effect has been reported previously in amorphous ferromagnets ${ }^{16}$ but, again, the actual mechanism is unclear.

We acknowledge stimulating discussions with Dr. H. Fukuyama and Dr. Y. Bruynseraede.

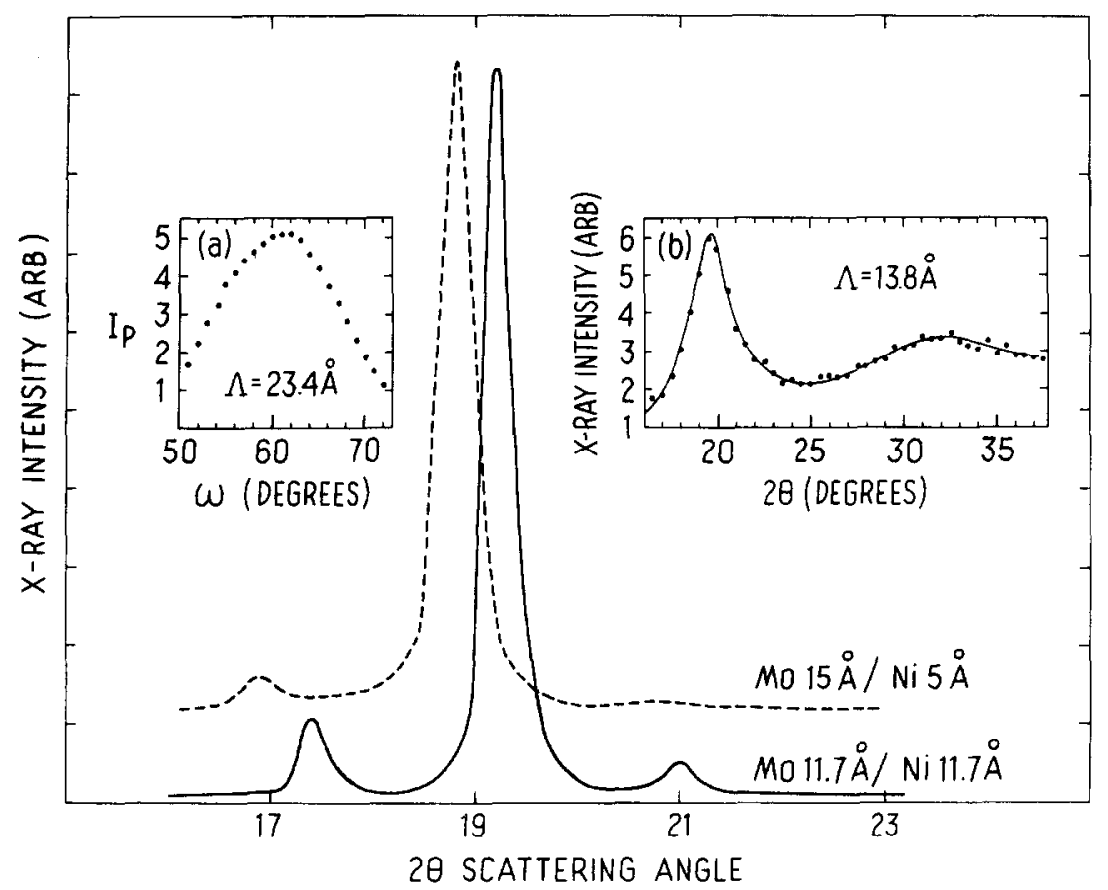

Fig. 4 Doe X-ray diffraction profiles for two samples showing coherent satellites. Inset (a): Rocking curve for Mo $11.7 \AA / N i$ 11.7A. Inset $(b)$ : Glassy behavior of Mo $6.9 A / N i 6.9 \AA$. 
The work was supported in part by NSF Low Temperature Grants DMR 8404975 (RC) and DMR 83-04356 (CU), by Grant

ARO DAAG-29-83-K-0131, by ONR Contract N00014-83-F-0031 and the U.S. Department of Energy.

\section{REFERENCES}

1. L.L. Chang and L. Esaki, Prog. Cryst. Growth Charact. 2, 3 (1979).

2. I.K. Schuller, Phys. Rev. Lett. 44, 1597 (1980).

3. M.R. Khan, C.S.L. Chun, G.P. Felcher, M. Grimsditch, A. Kueny, C.M. . Falco, and I.K. Schuller, Phys. Rev. B27, 7186 (1983).

4. M.R. Khan, P. Roach and I.K. Schuller, (to be pub1ished)

5. I.K. Schuller and A. Rahman, Phys. Rev. Lett. 50, 1377 (1983).

6. M.M. CoTiver and R.H. Hammond, Phys. Rev. Lett. 30, 92 (1973).

7. J. Geerk, M. Gurvitch, D.B. McWhan and
J.M. Rowe11, Physica 109/110B, 1775 (1982).

8. M. Hennion and B. Hennion, J. Phys. F8, 287 (1978).

9. R. Meservey, P.M. Tedrow, and V.R. Kalvey, J. App 1. Phys. 52, 1617 (1981).

10. Ding-Sheng Wang, A.J. Freeman, and $H$. Krakauer, Phys. Rev. B24, 1126 (1981).

11. J. Tersoff and L.M. FaTicov, Phys. Rev. B26, 6186 (1982).

12. I. Kramer and G. Bergmann, Phys. Rev. B27, 7271 (1983).

13. J.T. Masden and N. Giordano, Phys. Rev. B27, 6522 (1983).

14. B.L. Altshuler, D. Khmelnitskii, A.I. Larkin, and P.A. Lee, Phys. Rev. B22, 5142 (1980).

15. For an overview see articles in Percolation, Localization and Superconductivity, A.M. Goldman and S. Wolf, eds., NATO Advanced Study Institute, Les Arcs, France, 1983 (proceedings to be published).

16. R.W. Cochrane, F.T. Hedgcock, B.J. Kästner and W.B. Muir, J. de Phys. (Paris) 39 C6-939 (1978). 